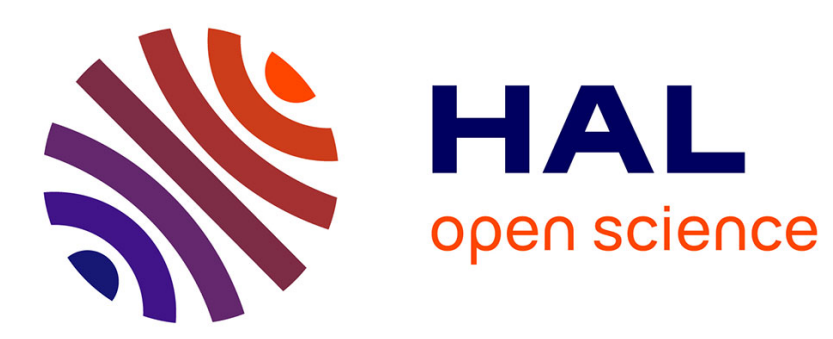

\title{
Les orateurs des municipes à Rome: intégration, réticences et snobismes
}

\author{
Jean-Michel David
}

\section{To cite this version:}

Jean-Michel David. Les orateurs des municipes à Rome: intégration, réticences et snobismes. Les "bourgeoisies" municipales italiennes aux IIe et Ier siècles av. J.-C., 1981, Paris, France. pp.309-323. hal-01056647

\section{HAL Id: hal-01056647 https://hal.science/hal-01056647}

Submitted on 20 Aug 2014

HAL is a multi-disciplinary open access archive for the deposit and dissemination of scientific research documents, whether they are published or not. The documents may come from teaching and research institutions in France or abroad, or from public or private research centers.
L'archive ouverte pluridisciplinaire HAL, est destinée au dépôt et à la diffusion de documents scientifiques de niveau recherche, publiés ou non, émanant des établissements d'enseignement et de recherche français ou étrangers, des laboratoires publics ou privés. 


\section{LES ORATEURS DES MUNICIPES À ROME: INTÉGRATION, RÉTICENCES ET SNOBISMES.}

Il ne suffit pas qu'un italien soit citoyen pour qu'il soit accepté. L'intégration dans la communauté ne se mesure pas seulement au statut juridique. Des notions plus fines interviennent et doublent la hiérarchie des catégories civiques d'un jeu subtil d'acceptation et de rejet. Les cercles aristocratiques surtout, n'accordent leur reconnaissance aux domi nobiles ambitieux qu'avec difficulté. Il leur imposent des procédures sévères d'évaluation de la conformité au modele dont ils sont les détenteurs légitimes. Ils pèsent tout: la naissance, le vêtement, le ton de la voix, l'élévation de la pensée, l'intelligence des situations... et marquent les distances - les distinctions - nécessaires à la reproduction de leur propre pouvoir en jouant de la condescendance ou du snobisme.

Toutes les sociétés connaissent la force et l'importance de ces pratiques (1). A Rome cependant, l'âpreté des conflits sociaux et politiques fait que ces nuances dáns l'échelle sociale deviennent autant de barrières à l'accès au pouvoir. Les magistratures, les commandements et les honneurs ne procèdent pas que du vote. Ils sont la contre-partie spontanée de la place que, de l'accord de tous, on tient dans la cité. C'est le regard critique que le groupe porte sur les conduites de chacun qui, avant toute procédure juridique, détermine les chances d'un individu à la reconnaissance collective. C'est dire l'importance des comportements oratoires qui mettent en vue, donnent à observer, à écouter et à juger.

L'éloquence judiciaire devient ainsi un bon moyen d'apprécier le degré d'intégration des domi nobiles à la communauté romaine. Elle ne nécessite aucune qualification juridique particulière: n'importe quel citoyen jouissant de ses capacités, a le droit d'en accuser ou d'en défendre un autre. Elle permet à tout inconnu de se faire des clients et d'acquérir la notoriété. Il n'est donc pas surprenant que, comme Cicéron, de nombreux jeunes italiens y aient investi leurs ambitions de petits notables soucieux de promotion. L'histoire littéraire devient ainsi un document précieux sur l'accueil que fit le public romain à ces nouveaux venus. Les jugements, les réticences et les applaudissements, le rire surtout, sont autant de critères qui permettent d'apprécier les formes de l'acceptation ou du refus.

Le Brutus, ce précieux dialogue sur l'éloquence, apporte des indications importantes. Cicéron y énumère en les jugeant, les orateurs qui l'ont précédé. Il y cite comme tels ceux qui viennent des villes d'Italie et surtout, les regroupe. Or les listes qu'il donne sont - on le sait - classées par ordre chronologique (2). Il faut donc que l'origine municipale entraîne une différence sensible de style ou de comportement pour que Cicéron interrompe sa classification et mette ces personnages de côté.

La première série est celle des paragraphes 167 à 172 . C'est la plus importante. Elle constitue un véritable excursus à l'intérieur du dialogue puisque Cicéron y renverse l'ordre chronologique pour citer L. Papirius de Frégelles:

eiusdem fere temporis fuit eques Romanus C. Titius, qui meo iudicio eo pervenisse videtur quo potuit fere Latinus orator sine Graecis litteris et sine multo usu pervenire. huius orationes tantum argutiarum tantum exemplorum tantum urbanitatis habent, ut paene Attico stilo scriptae esse videantur.

(1) Cf. bien entendu, P. Bourdieu, La Distinction, critique sociale du jugement, Paris, 1979.

(2) Cf. en dernier lieu, G.V. Sumner, The orators in Cicero's Brutus, Prosopography and chronology, Toronto, 1973. 


\section{JEAN-MICHEL DAVID}

easdem argutias in tragoedias satis ille quidem acute sed parum tragice transtulit. quem studebat imitari $L$. Afranius poeta, homo perargutus, in fabulis quidem etiam, ut scitis, disertus. fuit etiam $Q$. Rubrius Varro, qui a senatu hostis cum C. Mario iudicatus est, acer et vehemens accusator, in eo genere sane probabilis. doctus autem Graecis litteris propinquus noster, factus ad dicendum, M. Gratidius, $M$. Antoni perfamiliaris, cuius praefectus cum esset in Cilicia est interfectus, qui accusavit C. Fimbriam, M. Mari Gratidiani pater.

Atque etiam apud socios et Latinos oratores habiti sunt $Q$. Vettius Vettianus e Marsis, quem ipse cognovi, prudens vir et in dicendo brevis; $Q$. D. Valerii Sorani, vicini et familiares mei, non tam in dicendo admirabiles quam docti et Graecis litteris et Latinis; $C$. Rusticelius Bononiensis, is quidem et exercitatus et natura volubilis; omnium autem eloquentissimus extra hanc urbem T. Betutius Barrus Asculanus, cuius sunt aliquot orationes Asculi habitae; illa Romae contra Caepionem nobilis sane, quoi orationi Caepionis ore respondit Aelius, qui scriptitavit orationes multis, orator ipse numquam fuit. apud maiores autem nostros video disertissimum habitum ex Latio L. Papirium Fregellanum Ti. Gracchi P.f. fere aetate; eius etiam oratio est pro Fregellanis colonisque Latinis habita in senatu.

Les autres séries sont moins homogènes et en tout cas beaucoup plus courtes. Au paragraphe 180, on ne voit apparaître que Q. Sertorius et C. Gargonius:

sed omnium oratorum sive rabularum, qui et plane indocti et inurbani aut rustici etiam fuerunt, quos quidem ego cognoverim, solutissimum in dicendo et acutissimum iudico nostri ordinis $Q$. Sertorium, equestris C. Gargonium.

Aux paragraphes 241-242, les noms de L. Octavius, d'Aelius Staienus et des frères Caepasii, suivent ceux d'orateurs de style popularis:

et L. Octavius Reatinus, qui cum multas iam causas diceret, adulescens est mortuus - is tamen ad dicendum veniebat magis audacter quam parate - et $C$. Staienus, qui se ipse adoptaverat et de Staieno Aelium fecerat, fervido quodam et petulanti et furioso genere dicendi: quod quia multis gratum erat et probabatur, ascendisset ad honores, nisi in facinore manifesto deprehensus poenas legibus et iudicio dedisset. Eodem tempore C.L. Caepasii fratres fuerunt, qui multa opera, ignoti homines et repentini, quaestores celeriter facti sunt, oppidano quodam et incondito genere dicendi.

Ils précèdent ceux de $\mathrm{C}$. Cosconius Calidianus et de $\mathrm{Q}$. Arrius que l'on serait tenté de retenir si ce n'était que rien ne signale leur origine municipale.

Au paragraphe 271 enfin, apparaissent deux chevaliers romains de Spolète et de Pisaurum:

itaque ne hos quidem equites Romanos amicos nostros, qui nuper mortui sunt, <omittam>, $P$. Cominium Spoletinum, quo accusante defendi C. Cornelium, in quo et compositum dicendi genus et acre et expeditum fuit; T. Accium Pisaurensem, cuius accusationi respondi pro A. Cluentio, qui et accurate dicebat et satis copiose. eratque praeterea doctus Hermagorae praeceptis.

Un tableau d'ensemble permet de mieux saisir l'image que par leur style oratoire, ces personnages ont pu dégager d'eux-mêmes, ou plutôt celle que Cicéron a établie ou recueillie de leurs contemporains ou successeurs.

$\begin{array}{lll}\text { Nom } & \text { Origo } & \text { Jugements } \\ \text { C. Titius, } R E, \mathrm{n}^{\circ} 7 ; \text { ORF, } & ?(3) & \\ \mathrm{n}^{\circ} 51 \text { Sumner, } \mathrm{n}^{\circ} 112 & \end{array}$

(3) Les Titii sont nombreux et rien ne permet d'identifier l'origo de celui-ci. Le terme latinus orator sous lequel Cicéron le désigne s'oppose à l'éducation grecque qu'il n'avait pas reçue (sine graecis litteris et sine multo usu pervenire). De toutes façons, il ne peut être de droit latin car précédant $Q$. Rubrius Varro de Casinum et M. Gratidius d'Arpinum dans l'énumération, il est comme eux, citoyen romain. Bien sûr, rien n'exclut une origine municipale, mais il faut qu'il ait été très intégré dans la vie politique romaine pour pouvoir prononcer une suasio legis Fanniae (cf. C. Cichorius, Untersuchungen zu Lucilius, Berlin, 1908,
pp. 264-267, à mon sens toujours valable). 
LES ORATEURS DES MUNICIPES A ROME

Q. Rubrius Varro, $R E, \mathrm{n}^{\circ}$

24; Sumner, $\mathrm{n}^{\circ} 113$.

M. Gratidius, $R E, \mathrm{n}^{\circ} 2$;

$O R F, \mathrm{n}^{\circ}$ 54; Sumner, $\mathrm{n}^{\circ}$

114.

Q. Vettius Vettianus, $R E$, $\mathrm{n}^{\circ} 18$; Sumner, $\mathrm{n}^{\circ} 115$.

Q.D. Valerii, $R E, \mathrm{n}^{\circ} 54$ \&

345; Sumner, $n^{\circ} 116-117$.

C. Rusticelius, $R E, \mathrm{n}^{\circ} 2$;

Sumner $n^{\circ} 118$.

T. Betutius Barrus, $R E, \mathrm{n}^{\circ}$

1; ORF, $\mathrm{n}^{\circ} 84$; Sumner $\mathrm{n}^{\circ}$ 119.

L. Papirius, $R E, \mathrm{n}^{\circ} 19$; $O R F, \mathrm{n}^{\circ} 11 ;$ Sumner, $\mathrm{n}^{\circ}$ 120.

T. Tinca, $R E$, s.v. Tinca; Sumner, $\mathrm{n}^{\circ} 121$.

Q. Sertorius, $R E, \mathrm{n}^{\circ} 3$; $O R F, \mathrm{n}^{\circ}$ 81; Sumner, $\mathrm{n}^{\circ}$ 140.

C. Gargonius, $R E, \mathrm{n}^{\circ} 4$; Picenum, Campanie Sumner $\mathrm{n}^{\circ} 141$. $\quad \because \quad$ ou Ombrie (6)

L. Octavius, $R E, \mathrm{n}^{\circ} 28 ; \quad$ Reatinus

Sumner, $\mathrm{n}^{\circ} 188$.

C. Staienus, $R E, \mathrm{I}, \mathrm{n}^{\circ} 98$, col. 525; IIIa, col. 21332136; Sumner, $\mathrm{n}^{\circ} 189$.
Casinum (4)

Arpinum

e Marsis

Sorani (5)

\section{Bononiensis}

Asculanus

Fregellanus

Placentinus

Nursia

$?(7)$ acer et vehemens accusator, in eo genere sane probabilis,

doctus Graecis litteris... factus ad dicendum

prudens vir et in dicendo brevis

non tam in dicendo admirabiles quam docti et Graecis litteris et Latinis; De Or., III, 43: ex istis... urbanis, in quibus minimum est litterarum, nemo est quin litteratissimum togatorum omnium $Q$. Valerium Soranum levitate vocis atque ipso pressu et sono facile vincat. et exercitatus et natura volubilis

omnium autem eloquentissimus extra hanc urbem

facetissimus; Quint., I, 5, 12: nam duos in uno nomine faciebat barbarismos si reprehendenti Hortensio credimus.

omnium oratorum sive rabularum, qui et plane indocti et inurbani aut rustici etiam fuerunt ... solutissimum in dicendo et acutissimum iudico nostri ordinis $Q$. Sertorium, equestris $C$. Gargonium.

ad dicendum veniebat magis audacter quam parate.

fervido quodam et petulanti et furioso genere dicendi; cf. Pro Clu., 70; 72.

(4) Sur les Rubrii de Casinum, cf. T.P. Wiseman, New Men in the Roman Senate, Oxford, 1971, p. 257; CIL X, 5169; 5419; cf. aussi l'épitaphe d'origine et de date inconnue du jeune M. Rubrius Varro, CIL VI, 25528.

(5) L'identification que C. Cichorius, Zur Lebensgeschichte des Valerius Soranus, Hermes, 41, 1906, pp. 59-68, puis F. Della Corte, Per l'identità di Valerio Edituo con Valerio Sorano, Riv. Filol., 14, 1935, pp. 68-70; E. Gabba, Le origini della guerra sociale, Athenaeum, 32, 1954, p. 102, n. 1 et T.P. Wiseman, New Men, ${ }^{\circ}$ 457, p. 269, font avec Valerius Aedituus et l'érudit Q. Valerius mis à mort par Pompée (Plut., Pomp., 10,8) reste très tentante malgré les réserves de Sumner, Orators, p. 101.

(6) T.P. Wiseman, New Men, $\mathrm{n}^{\circ}$ 195, p. 235, Ombrie? et C. Nicolet, L'ordre équestre à l'époque républicaine, Paris, 1974, II, $\mathrm{n}^{\circ} 167$, p. 896 qui cite ILLRP, 304: Hadria; 714: Campanie.

(7) On ne connaît pas son origine de toutes façons assez humble (cf. Cic., Pro Clu., 87). T.P. Wiseman, New Men, p. 262, 
C.L. Caepasii, $R E$, s.v. Italie du Sud (8) Caepasius; $O R F, \mathrm{n}^{\circ} 115-$ 116; Sumner $n^{\circ} 190-191$.

P. Cominius, $R E \mathrm{n}^{\circ} 4 ; 8$; $O R F, \mathrm{n}^{\circ} 143$; Sumner, $\mathrm{n}^{\circ}$ 214.

T. Accius, $R E$, Sup. I, $\mathrm{n}^{\circ}$ $1 \mathrm{a} ; O R F, \mathrm{n}^{\circ} 145$.
Spoletinus

Pisaurensis ignoti homines et repentini... oppidano quodam et incondito genere dicendi; cf. Pro Clu., 57.

compositum dicendi genus et acre et expeditum fuit.

et accurate dicebat et satis copiose, eratque praeterea doctus Hermagorae praeceptis; Pro Clu., 156: bonus et disertus.

Grâce au Brutus, on dispose ainsi d'un échantillon à peu près homogène d'orateurs d'origine municipale. C'est ce critère en effet qui les isole du reste de l'énumération chronologique. Les jugements que Cicéron porte sur leur style oratoire ont donc toutes les chances d'associer origine et comportement et donc d'être caractéristiques de l'image du domi nobilis tentant son intégration à Rome.

On distingue ainsi deux séries d'appréciations. Les unes portent sur des qualités d'intelligence ou de comportement: la richesse d'invention, la force ou la véhémence de l'action oratoire; les autres sur des qualités de naissance ou de formation: la connaissance du grec ou des préceptes, le ton ou le son de la voix.

Presque toutes les premières renvoient à des formes typiques de l'eloquentia popularis (9): acer et vehemens accusator (Q. Rubrius Varro), rabula (Q. Sertorius et C. Gargonius) ad dicendum veniebat magis audacter quam parate (L. Octavius) fervido quodam et petulanti et furioso genere dicendi (C. Aelius Staienus), compositum dicendi genus et acre et expeditum (P. Cominius) ou à des formes atténuées mais qui impliquent une action oratoire animée: et exercitatus et natura volubilis (C. Rusticelius), solutissimus in dicendo et acutissimus (Q. Sertorius et C. Gargonius), et accurate dicebat et satis copiose (T. Accius). La seule exception véritable est représentée par Q. Vettius Vettianus: prudens vir et in dicendo brevis.

Un tel lien entre l'origine municipale et l'eloquentia popularis, même s'il n'est pas surprenant, mérite qu'on cherche à l'expliquer. Je l'avais déjà remarqué dans un article précédent, mais c'était en étudiant l'accusation populaire. A partir des Gracques, l'élargissement du droit pour tous d'accuser un sénateur coupable de malversations, permettait à des orateurs venus de toutes les villes d'Italie, de se faire une place sur le Forum de Rome. Dans une certaine mesure, le fait de prendre publiquement la parole, pour la première fois dans une procédure de iudicium publicum, les mettait dans une situation comparable à celle des homines novi. Ils étaient donc contraints à une éloquence agressive qui correspondait à la fois à la nécessité pour un accusateur de suscitare invidiam et à leur position de challenger face à un aristocrate sûr de la légitimité d'un pouvoir hérité et reconnu (10).

cite un centurion L. Staienus au CIL VI, 3600; Münzer, RE, ad loc., indique que le gentilíce Staius est osque. Il est invraisemblable cependant qu'il soit passé dans la gens Aelia par adoption fictive. Cicéron indique en effet qu'il prit le cognomen Paetus pour s'identifier aux Aelii Paeti. Il fallait donc qu'il fût déjà un Aelius pour que cette usurpation eut des chances d'être efficace. Toutefois, une origine municipale reste envisageable. Le mot de Cicéron hoc (Paetus) enim sibi Staienus cognomen ex imaginibus Aeliorum delegarat ne, si se Ligurem fecisset, nationis magis suae quam generis uti cognomine videretur. 'isse au moins imaginer, même s'il ne prouve évidemment pas qu'il était réellement d'origine ligure.

(8) C'est l'hypothèse de C. Nicolet, $O E$, II, p. 818.

(9) «Eloquentia popularis» et conduites symboliques des orateurs de la fin de la République: problèmes d'efficacité, QS, 12, 1980, pp. 171-211.

(10) Promotion civique et droit à la parole: L. Licinius Crassus, les accusateurs et les rhéteurs latins, MÉFRA, 91, 1979, pp. 135-181, en part., p. 156. 


\section{LES ORATEURS DES MUNICIPES A ROME}

Cette forme d'intervention oratoire n'est attestée que pour Q. Rubrius Varro (11), M. Gratidius (12), T. Betutius Barrus (13), P. et C. Cominius (14) et T. Accius (15). Mais notre documentation est très lacunaire et l'on ne sait pas grand-chose de l'activité des autres orateurs. Il est très possible qu'ils aient participé à une accusation devant une quaestio sans que nous n'en sachions rien. C'était là, en effet, un des moyens les plus accessibles à qui voulait se faire une place dans la classe politique de Rome.

Mais ne réduisons pas! Le choix de l'eloquentia popularis correspond aussi à une situation plus générale. Un bon nombre de ceux qui viennent ainsi de leurs municipes, apparaissent isolément sans l'appui du groupe serré d'amis qui protège et assure à l'inconnu la confiance du public. Cicéron dit bien que les frères Caepasii étaient des ignoti homines et repentini (16) et ajoute dans le Pro Cluentio que c'est inopia et necessitate coactus que Fabricius... ad Caepasios fratres confugit, homines industrios atque eo animo ut quaecumque dicendi potestas esset data in honore atque in beneficio ponerent (17). Asconius rappelle surtout que lorsque en 66 , les frères Cominii accusèrent $\mathrm{C}$. Cornelius le tribun de 67, ils furent entourés par les amis de leur adversaire, menacés de mort et contraints à la fuite (18). Jamais, les uns ou les autres, s'ils s'étaient intégrés dans un solide réseau clientélaire, n'auraient eu à subir de tels jugements ou de telles mésaventures.

Accusateurs téméraires ou gagne-petit de la reconnaissance, ces orateurs d'origine municipale interviennent directement sur le Forum de Rome. Ils n'attendent pas d'avoir reçu l'agrément de l'aristocratie et évitent ainsi l'itinéraire compliqué de l'intégration à ses clientèles. Ils ne recherchent pas la caution que sa légitimité pourrait leur donner et refusent le rapport de subordination qu'ils devraient accepter en échange. Comme Marius lorsqu'il se présenta au consulat (19), ils cherchent à gagner directement la faveur populaire et adoptent avec l'eloquentia popularis, un comportement qui s'adapte à cette fin (20). Ils rompent ainsi avec les modèles de mesure et de refus du pathétique d'une aristocratie soucieuse de ne pas mettre en jeu l'autorité héréditaire que lui accorde la reconnaissance publique. Ils s'épargnent ainsi les coûteux investissements sociaux et symboliques qu'eût exigé le droit de ne prendre la parole en public qu'après avoir obtenu le statut que la tradition rendait nécessaire.

Ce rapport entre norme sociale et style oratoire est d'une extrême importance. Il explique que la réaction aristocratique à l'accusation populaire et à l'eloquentia popularis ait pris l'aspect d'un rejet

(11) cf. ibid., p. 179.

(12) cf. ibid., p. 177

(13) cf. ibid., p. $176 . \quad \cdot$

(14) En 74-73, ils accusent et font condamner C. Aelius Staienus de maiestate (Cic., Pro Clu., 100; 102; cf. 2 Verr., II, 79; Ps. Ascon., p. 219 St.; Schol. Pers., Sat., 2, 19; et aussi Cic., Top., 75; E.S. Gruen, The Last Generation of the Roman Republic, Berkeley \& Los Angeles, 1974, p. 524). Ils réapparaissent en 66, quand ils tentent d'accuser C. Cornelius de maiestate. Menacés de mort, par les amis de leur adversaire, ils s'enfuient. En 65, P. Cominius revient à la charge mais échoue (Ascon., p. 59-60; 61; 81 C.; cf. R.A. Bauman, The Crimen Maiestatis, Johannesbourg, 1967, pp. 71-75; A.M. Ward, Politics in the trials of Manilius and Cornelius, TAPhA, 101, 1970, p. 554-556; Gruen, o.c., p. 263-265; M. Griffin, The tribune C. Cornelius, JRS, 63, 1973, pp. 196-213, en part. 211-213).

(15) Il intervient aux côtés du fils d'Oppianicus dans l'accusation de sicariis et veneficiis qu'ils portent contre A. Cluentius Habitus en 66. Mais il est le seul à plaider vraiment. Il faut donc le considérer comme un quasi-professionnel (Cic., Pro Clu., 62; $65 ; 156-157 ; 160 ; 186 ;$ cf. $84 ; 86 ; 100 ; 135 ; 138 ; 143 ; 147-150$; sur le fond de l'affaire, cf. G. Hoenigswald, The murder charge in Cicero's Pro Cluentio, TAPhA, 93, 1962, pp. 109-123; E. Van Ooteghem, L'affaire Cluentius, Mel. Renard, II, 1969, pp. 777-788 et surtout C.J. Classen, Die Anklage gegen A. Cluentius Habitus, ZRG, 89, 1972, pp. 1-17).

(16) Br., 242.

(17) Pro Clu., 57; deterrimus obscurissimusque patronus (ibid.).

(18) Ascon., p. 59-60 C.: circumventi sunt ante tribunal eius accusatoris a notis operarum ducibus ita ut mors intentaretur, si mox non desisterent. Quam perniciem vix effugerunt interventu consulum qui advocati reo descenderant.

(19) cf. MEFRA, 91, 1979, p. 155.

(20) cf. $Q S, 12,1980$, pp. 182-183. 
violent de formes d'apprentissage ou de comportements oratoires. Les mots que Cicéron emploie ici, en sont l'expression. Ils sont caractéristiques du vocabulaire d'invective qui dénonce et qui exclut. J'ai déjà eu l'occasion de les étudier ailleurs, je n'y reviendrai pas (21). Je m'arrêterai davantage sur les jugements qui évaluent le degré de formation rhétorique ou d'élégance de l'expression. On y retrouve spontanément l'association du raffinement et du style oratoire.

Ceux qui ont adopté l'eloquentia popularis, sont en même temps les plus mal formés: $Q$. Sertorius et C. Gargonius, plane indocti et inurbani aut rustici; L. Octavius magis audacter quam parate; $\mathrm{C}$. et L. Caepasius, oppidano quodam et incondito genere dicendi. Le lien n'est pas fortuit; le modèle éducatif traditionnel passe aussi par une intégration aux relations clientélaires. L'exemple de Cicéron le prouve (22). C'est au cours du tirocinium fori que s'opère l'apprentissage simultané des règles de la rhétorique et des normes de conduite en public. C'est en s'agrégeant à l'entourage d'un aristocrate que l'on apprend, en l'imitant, à parler et à se comporter. Mais c'est ainsi aussi que l'on se subordonne publiquement à son jugement et à son autorité. On peut certes apprendre les règles de l'éloquence auprès des maîtres de rhétorique. Mais, à partir de 92 a.C., leur enseignement est interdit (23); et même s'il ne l'était pas, ils ne pourraient apporter la caution sociale que donne l'intégration au sein de la classe politique romaine.

Or cette intégration reste difficile pour ceux qui viennent des municipes. Il ne suffit pas en effet de vouloir être admis, encore faut-il être reçu. Et, les jugements que Cicéron porte sur les meilleurs, le montrent bien: il leur manque l'urbanitas.

Nostri minus student litteris quam Latini. Tamen ex istis, quos nostis, urbanis, in quibus minimum est litterarum, nemo est quin litteratissimum togatorum omnium $Q$. Valerium Soranum leuitate uocis atque ipso oris pressu et sono facile uincat (24).

ou plus précisément, ce passage essentiel du Brutus qui suit la première énumération des orateurs d'origine municipale (25),

Tum Brutus: quid tu igitur, inquit, tribuis istis externis quasi oratoribus?

Quid censes, inquam, nisi idem quod urbanis? praeter unum, quod non est eorum urbanitate quadam quasi colorata oratio.

Et Brutus: qui est, inquit, iste tandem urbanitatis color?

Nescio, inquam; tantum esse quendam scio. id tu, Brute, iam intelleges, cum in Galliam veneris; audies tu quidem etiam verba quaedam non trita Romae, sed haec mutari dediscique possunt; illud est maius, quod in vocibus nostrorum oratorum retinnit quiddam et resonat urbanius. nec hoc in oratoribus modo apparet, sed etiam in ceteris. ego memini T. Tincam Placentinum hominem facetissimum cum familiari nostro $Q$. Granio praecone dicacitate certare.

Eon', inquit Brutus, de quo multa Lucilius?

Isto ipso; sed Tincam non minus multa ridicule dicentem Granius obruebat nescio quo sapore vernaculo; ut ego iam non mirer illud Theophrasto accidisse, quod dicitur, cum percontaretur ex anicula quadam quanti aliquid venderet et respondisset illa atque addidisset 'hospes, non pote minoris', tulisse eum moleste se non effugere hospitis speciem, quom aetatem ageret Athenis optumeque loqueretur omnium. sic, ut opinor, in nostris est quidam urbanorum sicut illic Atticorum sonus. sed domum redeamus, id est ad nostros revortamur.

(21) MÉFRA, 91, 1979, pp. 156-157; QS, 12, 1980, pp. 181-182.

(22) Sur l'éducation de Cicérón et son intégration clientélaire, cf. en dernier lieu, K.A. Neuhausen, Ciceros Vater, der Augur Scävola und der junge Cicero, WS, 92, 1979, pp. 76-87.

(23) MEFRA, 91, 1979, pp. 156-160; 169.

(24) De Or., III, 42-43.

(25) Br., 170-172. 


\section{LES ORATEURS DES MUNICIPES A ROME}

L'urbanitas est une notion difficile à saisir car elle est chargée de plusieurs valeurs sémantiques. Elle signifie d'abord ce qui est propre à Rome; urbani, comme dans ce dernier texte ( $\$ 170)$, signifie les gens de la Ville. Mais elle signifie en même temps les manières, la langue et le goût de Rome, le sens de l'humour, et rejoint aussi la notion stoïcienne d" $\alpha \sigma \tau \varepsilon i ̈ \mu$ ós. On a beaucoup étudié les rapports qui lient, d'un auteur à l'autre, ces diverses significations (26). Il n'est pas nécessaire d'y revenir ici. Essayons simplement, en suivant Cicéron, d'examiner concrètement ce dont sont dépourvus ces malheureux italiens.

Leur premier défaut est de mal prononcer le latin. Q. Valerius de Sora est un érudit raffiné, mais le premier romain venu l'emporte sur lui par la légèreté de la voix et le modelé du son. Il faut donc croire qu'il existait une façon de prononcer spécifiquement romaine, faite de nuances insensibles et inaccessibles à qui n'avait pas passé sa petite enfance à Rome même. C'est ce qu'indique cette notion si imprécise d'urbanitatis color (27) que Cicéron ne peut définir que négativement. On ne la perçoit qu'en quittant Rome et en sentant, par exemple en Gaule Cisalpine, les différences de prononciation ou d'accentuation du latin, plus caractéristiques encore que l'emploi d'un mot peu courant. La comparaison avec Théophraste finit d'éclairer la difficulté (28). Celui qui n'est pas né dans une ville, qui n'y a pas appris à parler, ne peut en avoir acquis l'accent (29). Le son de la voix opère ici la fonction de distinction.

Il est évident que personne ne peut, même en y séjournant longtemps, avoir assimilé tous les idiotismes et surtout, toutes les nuances d'intonation du latin de Rome. Commettre une faute en la matière, c'est alors se laisser identifier comme étranger (30). Ce ne serait rien si l'on ne désirait au contraire se fondre dans la communauté romaine. Cette nuance, ou plutôt cet écart, parce qu'il est à la fois insurmontable et insupportable, devient alors décisif dans la procédure de promotion et d'intégration sociale des domi nobiles.

Le sens de l'humour est l'autre aspect de l'urbanitas que Cicéron oppose aux orateurs des municipes. Il rappelle en effet cette joute oratoire où $\mathrm{Q}$. Granius, le praeco, rivalisant de plaisanteries avec T. Tinca de Plaisance (31), l'emporta sur son adversaire. Curieusement, cet argument s'insère dans le développement sur l'urbanitas-son de la voix et ce n'est pas l'association d'idées qui lie T. Tinca à la Gaule Cisalpine qui peut l'expliquer. Pour Cicéron, les plaisanteries de Granius doivent leur

(26) Cf. O. Lutsch, Die Urbanitas nach Cicero, Festgabe für W. Crecelius, Elberfeld, 1881, pp. 80-95, qui fait un bilan de ces différentes valeurs; H. Bléry, Rusticité et urbanité romaine, Paris, 1909 (sans intérêt, mélange de lieux communs sur la «civilisation» des Romains et_de paraphrases d'auteurs anciens); E. Frank, De vocis urbanitas apud Ciceronem vi atque usu,

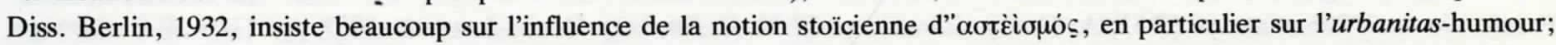
E. De Saint-Denis; Evolution sémantique de «urbanus-urbanitas», Latomus, 1939, pp. 5-24, qui croit pouvoir discerner la chronologie de l'évolution sémantique: l'urbanitas-politesse n'apparaîtrait qu'au Ier s.; et surtout E.S. Ramage, Early Roman Urbanity, AJPh., 81, 1960, pp. 65-72; Urbanitas, Cicero and Quintilian, a contrast in attitudes, AJPh, 84, 1963, pp. 390-414; Ciceron on extra Roman speech, TAPhA, 92, 1961, pp. 481-494; Urbanitas. Ancient Sophistication and refinement, Cincinnati, 1973, qui insiste à très juste titre sur le fait que l'urbanitas chez Cicéron est une attitude d'exclusivisme à l'égard des autres qui s'appuie sur des qualités naturelles que l'on ne peut acquérir (cf. en part. AJPh., 84, pp. 390; 393; 403; Urbanitas, p. 5; 55). Cf. aussi A. Haury, L'ironie et l'humour chez Cicéron, Paris, 1955, pp. 19; 55-59.

(27) Cf. Cic., De Or., III, 161: nam et odor urbanitatis et mollitudo humanitatis et murmur maris et dulcitudo orationis sunt ducta a ceteris sensibus.

(28) Cf. O. Lutsch, Festg. Crecelius, pp. 82-83; E. De Saint-Denis, Latomus, 1939, p. 19; A.H. Travis, Parallels to Patavinitas, CP, 48, 1953, pp. 174-175; et surtout E.S. Ramage, TAPhA, 92, 1961, pp. 481-494; AJPh, 84, 1963, pp. 400-403; Urbanitas, pp. 59-62.

(29) Sur l'importance de l'apprentissage d'une langue correcte au cours de la petite enfance, cf. Cic., Br., 258; Quint., I, 1, 4-6.

(30) A l'inverse une prononciation rustique est, de la part d'un Romain authentique, un signe d'affectation. Cicéron en fait le reproche à Aurelius Cotta (De Or., III, 42).

(31) On ne le connaît que par ce passage. Tinca-Tinga est un cognomen celtique, cf. Münzer, RE, VIa, col. 1372, 1936. 


\section{JEAN-MICHEL DAVID}

meilleure efficacité à nescio quo sapore vernaculo. Il ne peut s'agir seulement de l'intonation avec laquelle Granius parlait. Ses plaisanteries devaient avoir un sel particulier qui résidait dans les énoncés eux-mêmes. Mais alors quel lien avec le son de la voix qui relève de l'énonciation?

Il ne peut être que dans la connivence, qui lie tous ensemble les membres de la communauté romaine, public de la scène. De même que l'accent n'est pas autre chose qu'un contexte de l'énonciation qui associe tous ceux qui le partagent, la plaisanterie n'a de sens que dans un contexte social tellement riche et complexe que la connaissance n'en est accessible qu'à ceux -qu'unit une longue familiarité. Le raisonnement n'y a pas de place. Le rire est l'effet d'une surprise, d'une énigme qui se résout dans l'intuition d'un sens caché. Il faut donc que le code que tous les participants partagent spontanément, soit très largement inexplicite pour que l'effet d'intrigue puisse opérer (32). Seul le partage d'une culture commune dans toute son étendue peut permettre le plaisir de la finesse. Nul doute que l'orateur issu d'un municipe ne soit vite perdant à ces compétitions et n'y révèle sa réelle origine. L'urbanitas-plaisanterie est donc elle aussi un facteur de distinction (33). Comme pour la prononciation du latin, elle est un écart révélateur. Mais il est d'autant plus insurmontable cette fois, qu'à la différence d'un accent qui évolue peu, la culture commune change constamment et que le snobisme a pour effet d'inventer de nouvelles subtilités qui augmentent encore la distance à parcourir.

Cette inégalité devant l'humour invite à poursuivre. Les orateurs d'origine municipale, si défavorisés dans l'emploi de la plaisanterie, n'en seraient-ils pas aussi les victimes désignées? Il faudrait tenter une sorte de prosopographie de la moquerie en public et profiter des quelques recueils de bons mots dont on dispose (34), pour faire l'essai d'un sondage.

Le premier, le plus important aussi parce qu'il est contemporain de notre période, est celui que Cicéron propose dans le De Oratore (35). C. Iulius Caesar Strabo y donne en effet quelques conseils sur l'emploi du rire dans l'éloquence et apporte quelques exemples de plaisanteries, sortes de duels où deux individus s'opposent en public. Certes les non-romains n'y apparaissent pas plus souvent que les autres puisqu'on ne peut compter comme tels que L. Aelius Lamia (36) (\$§ 262; 269), Helvius Mancia (37) (§ 266), Septimuleius d’Anagni (38) (§ 269) et M. Antistius de Pyrgi (39) (§ 287). Cette faible

(32) Cf. en particulier M. Hodgart, La Satire, Paris, 1969, pp. 107-108.

(33) Sur l'association urbanitas et sens de l'humour, cf. Cicéron qui indique très explicitement que, moins qu'ailleurs, il ne saurait y avoir de préceptes en matière de rire, De Or., II, 216: quae etiam si alia omnia tradi arte possunt, propria certe neque ullam artem desiderant; cf. 217; 220; 229; 232; et aussi Br., 143; 177; De Or., I, 17; 159; II, 217; 227; 228; 231; 269; De Fin., I, 7 ; 39; II, 103; De Off., I, 104; De Dom., 92; Pro Cael. 6; Fam., VII, 32, 2-3; IX, 15, 2; O. Lutsch, Festg. Crecelius, p. 85-91; E. Frank, De vocis urbanitas, p. 37-51; A. Haury, L'ironie, p. 57-59; E. De Saint-Denis, Latomus, 1939, pp. 11-14; E.S. Ramage, AJPh, 84, 1963, pp. 396-399; Urbanitas, pp. 56-59.

(34) Sur les recueils de bons mots, cf. outre les ouvrages cités n. 35, H. Peter, Die Litteratur des Witzwortes in Rom und die geflügelten Worte im Munde Caesars, NJPhP, 155, 1897, pp. 853-860, en part., pp. 853-856.

(35) II, 216-290; cf. A. Plebe, La teoria del comico da Aristotele a Plutarco, Turin, 1952, pp. 64-75; et surtout, A. Michel, Rhétorique et Philosophie chez Cicéron, Paris, 1960, pp. 271-283; A. Manzo, Dalla teoria alla prassi del faceto in Cicerone. Rend.Ist.Lomb.Cl.Lett., 100, 1966, pp. 397-411 et G. Monaco, ed. Cicéron, L'excursus de ridiculis (De Or., II, 216-290), Palerme, 1968, en part., pp. 22-28: sur les sources de Cicéron et sa conception du comique.

(36) Cf. Klebs, $R E, \mathrm{I}, \mathrm{n}^{\circ} 74$, col. 522, 1893. L'anecdote donne l'impression qu'Aelius Lamia relativement jeune encore, est écrasé par la maturité de Crassus. Ce procès devrait donc se situer dans la première décennie du Ier siècle, mais il est impossible de le dater avec plus de précision (cf. $O R F^{4}$, p. 253, ad n ${ }^{\circ} \mathrm{XI}$ ). Aelius Lamia est probablement le père du préteur de 42 (cf. Nicolet, $O E$, II, p. 764, n. 3) et donc aussi un ami de Cicéron (Pro Sest., 29). Comme son client, Marius Gratidianus, et son adversaire, Visellius Aculeo, il appartient donc sans doute à ce milieu équestre d'origine municipale (Arpinum et Campanie) proche des Tullii (cf. De Off., III, 67; De Or., II, 2).

(37) Cf. Münzer, $R E$, VIII, 1, $\mathrm{n}^{\circ} 15$, col. 229, 1912; $O R F^{4}, \mathrm{n}^{\circ} 71$, p. 270-271; MEFRA, 91, 1979, p. 177; Val.Max., VI, 2, 8: Formianus.

(38) Cf. Münzer, $R E$, IIa, s.v. Septumuleius, coll. 1621-1622.

(39) Cf. Klebs, $R E, \mathrm{I}, \mathrm{n}^{\circ} 17$, col. 2547, 1896. 
fréquence n'a bien sûr aucune signification: on ne peut demander à un tel texte d'être un relevé statistique. Ce qui en revanche mérite d'êtré relevé, c'est que si les deux derniers sont victimes de jugements moraux, L. Aelius Lamia et Helvius Mancia subissent des railleries qui portent sur leur physique ou leur formation intellectuelle. Le dernier surtout est donné en exemple de moquerie par révélation de défauts corporels: valde autem ridentur etiam imagines, quae fere in deformitatem aut in aliquod vitium corporis ducuntur cum similitudine turpioris (40). De telles plaisanteries n'auraient aucun effet si leurs victimes n'étaient peu connues du public. C'est le manque de familiarité qui les rend possibles. T. Pinarius, l'autre exemple de moquerie physique n'est peut-être pas originaire d'un municipe, mais lui aussi est un inconnu (41). En fait, il manque à ces personnages d'être déjà des hommes publics au moment où ils prennent la parole. Ils éviteraient ainsi ces rires qui sont autant de rejets. Le malheureux Lamia a beau s'excuser en disant qu'il n'est pas responsable de son visage, Crassus lui refuse tout esprit. On naît romain, on ne le devient pas.

Les exemples de l'autre grand excursus consacré au rire, celui de Quintilien (42), confirment cette impression. Certes, il est beaucoup plus tardif, mais les anecdotes y sont presque toutes empruntées à Cicéron et l'on peut l'utiliser, au moins à titre de comparaison. On y retrouve Helvius Mancia (43) et Caepasius (44), et, à côté d'eux, M. Plaetorius (45), Tuccius (46), P. Vatinius (47) et Lartius (48). Or, si on laisse de côté les épisodes trop obscurs qui mettent en scène Tuccius et Lartius ainsi que celui qui concerne Caepasius et qui relève davantage d'un comique de situation, on constate que là encore, les plaisanteries portent sur la naissance (Plaetorius) ou l'aspect corporel (P. Vatinius). Autant de qualités ou de défauts dont les intéressés ne sont pas maîtres (49), et qui rendent. ces bons mots particulièrement brutaux.

Certes, la valeur statistique de ces recueils de plaisanteries est nulle. Mais le choix que Cicéron et Quintilien ont opéré, se veut exemplaire. Ces moqueries ont valeur de modèle; elles répondent donc à des normes sociales (50). Comment ne pas être frappé alors par le caractère nécessaire de cette liaison entre l'origine municipale ou plus simplement modeste de ceux dont on se moque et la propension à faire porter la plaisanterie sur l'aspect physique ou la naissance? La plupart des non-romains qui apparaissent dans ces anecdotes sont victimes de brocards de ce genre et, réciproquement, quand

(40) § 266; cf. 239.

(41) Münzer, $R E, \mathrm{XX}, 2, \mathrm{n}^{\circ} 7$, col. 1398, 1950. La gens est patricienne et a donné des magistrats aux V-IVème s., puis de nouveau à la fin du Illème et au début du Ilème s. Il est peut-être le père de l'autre T. Pinarius que l'on connait, le familiaris de Cicéron $\left(R E, \mathrm{n}^{\circ} 8\right)$.

(42) Inst. Or., VI, 3; cf. G. Monaco, ed. Quintiliano, Il capitolo de risu (inst. or. VI, 3), Palerme, 1970.

(43) VI, $3,38$.

(44) cf. supra. L'anecdote est celle que raconte Cicéron dans le Pro Cluentio, 58-59.

(45) M. Plaetorius Cestianus, Münzer, RE, XX, 2, n 16, coll. 1950-1952, 1950; Wiseman, New Men, n 320, p. 251. Il est certainement un Cestius de Préneste adopté par un Plaetorius de Tusculum, sans doute M. Plaetorius, tué sur ordre de Sylla en 82 .

(46) Sans doute M. Tuccius Galeo, cf. Münzer, $R E$, VIIa, 1, $\mathrm{n}^{\circ}$ 6, col. 767, 1939. A. Villius est incertain. Ce nom est assez répandu en Campanie (cf. ILLRP, 709; 735; 744; 745; cf. aussi 634 à Ostie) et on peut se demander s'il ne descend pas d'un des colons installés en 186 à Siponte ou à Buxentum par M. Tuccius, le préteur de 190.

(47) Cf. H. Gundel, RE, VIIIa, 1, n 3, coll. 495-520 avec L. Ross Taylor, VDRR, p. 262-263; Wiseman, New Men, $\mathrm{n}^{\circ} 467$, p. 270.

(48) Cf. Münzer, $R E$, XII, $\mathrm{n}^{\circ}$ 1, coll. 877-878, 1924; Wiseman, New Men, $\mathrm{n}^{\circ} 223$, p. 237, qui fait l'hypothèse d'une origine à Castrum Novum; cf. aussi les Larcii de Campanie: ILLRP, 745; 833; 977.

(49) Cf. aussi la plaisanterie dont est victime Sex. Clodius Phormio, sans doute un affranchi (VI, 3, 56).

(50) Une liste complète des plaisanteries à caractère corporel montrerait évidemment que les non-romains ne sont pas les seuls à être visés, cf. e.g. le «choix» de J.-P. Cèbe, La caricature et la parodie dans le monde romain antique, Paris, 1966, pp. 129-131 et les listes de J. Opelt, Die lateinischen Schimpfwörten und verwandte sprachliche Erscheinungen, Heidelberg, 1965, pp. $149-151 ; 153$. 
Cicéron ou Quintilien se mettent à traiter des plaisanteries sur l'aspect corporel (51), ce sont eux qu'ils mettent en scène. Reprocher son visage, son père ou sa mère à un non-romain est donc aussi une recette d'école, un lieu commun de l'humour oratoire. Or, le caractère radical de ces reproches saute aux yeux: rien ne peut modifier une naissance ou un visage. En cela, cet humour se rapproche de l'invective (52). Pires que les définitions outrageantes qui rejettent les orateurs de l'eloquentia popularis hors du cercle des gens bien élevés, ces bons mots contestent à leurs victimes le droit d'être des hommes: ils visent à rejeter et à exclure.

Mais ils ne peuvent anéantir complètement. Ceux qu'ils visent sont capables de réparties et la plaisanterie tourne vite au duel oratoire (53). Surtout, le mécanisme du rire est plus complexe que celui de la colère. Les plaisanteries du De Oratore sur Aelius Lamia et Helvius Mancia en sont de bons exemples. Dans la tension que crée l'altercation, l'attente des réparties se transforme brusquement en éclat de rire. Celui qui se moque, révèle une image de son adversaire que tous pressentaient sans la voir. En provoquant l'intuition d'un travers, il rend évidente la connivence qui le lie à son public. Il gagne la reconnaissance d'un groupe qui trouve dans le rire un plaisir d'autant plus partagé qu'il naît de l'inconscient du code. Celui dont on se moque, n'est pourtant pas complètement exclu. Comme le dit Cicéron, le rire désarme, il ne rend pas odieux (54). Il mutile ses victimes, il ne les tue pas. Par le plaisir qu'il donne, il retient et repousse à la fois, il écarte et met à distance.

Les orateurs qui viennent des municipes ont donc tout à perdre à l'emploi de plaisanteries. Moins efficaces que de vieux romains quand ils s'y essaient, plus durement touchés quand ils en sont victimes, ils y révèlent l'écart qui les sépare de la communauté romaine. Ne pas y être né, ne pas avoir longtemps vécu à Rome, interdit d'en partager le code dans toute sa profondeur. Faut-il donc s'étonner que lorsqu'ils prennent la parole, ils aient tendance à solliciter des sentiments plus universels de pitié ou de colère. L'émotion et le rire sont le plus souvent incompatibles (55). Ceux qui choisissent l'eloquentia popularis, déplacent le débat sur un terrain plus sûr, moins accessible en tout cas à l'ironie d'un adversaire assuré de sa place au sein du groupe civique.

Tout cela serait bien simple, si - étrangement - les moqueurs n'étaient pas eux-mêmes originaires de bourgades d'Italie. Cicéron en est le meilleur exemple. C'est lui, le fils de notables d'Arpinum, qui se moque de Plaetorius, de Sex. Clodius Phormio et de Vatinius. C'est lui qui écrit le De Oratore et rappelle avec complaisance les bons mots de Crassus ou de Caesar Strabo - des romains légitimes ceux-la - sur Aelius Lamia ou Helvius Mancia. N'est-ce pas paradoxal de la part d'un homme qui a eu lui-même à souffrir au cours de sa carrière du rappel injurieux de son origine municipale (56)?

Mais ne s'agirait-il que de Cicéron! Trois autres personnages méritent que l'on s'intéresse à eux: Q. Granius, Lucilius et Varron. Les deux premiers surtout, présentent en effet ce caractère étrange d'être à la fois d'origine municipale et des modèles d'urbanitas. Cicéron, par exemple, les cite lorsqu'écrivant à son ami Paetus, il regrette le temps heureux où Rome n'était pas envahie par les provinciaux amenés par César.

(51) On pourrait ajouter les deux apophtegmes cités par Plutarque qui portent sur le corps: ils visent $P$. Vatinius (8) et un Octavius originaire de Libye (9); cf. Plut., Cic., 26, 3; 5.

(52) Cf. e.g. le passage de Crassus de la plaisanterie à l'invective lorsqu'il plaide contre Iunius Brutus (De Or., II, 222-227; cf. A. Michel, Rhétorique et philosophie, pp. 287-288), et surtout R.C. Elliot, The power of satire, magic ritual, art, Princeton, 1960, qui associe la satire au pouvoir magique de négation ou de destruction par les mots, en part., pp. 49-99 et pp. 127-128 (sur les traces du malum carmen dans la satire latine classique).

(53) Cf. la boutade lancée par Helvius Mancia à Antoine, Cic., De Or., II, 274, et le duel déjà cité de Granius et de T. Tinca.

(54) De Or., II, 236: (Hilaritas) vel quod frangit adversarium, quod impedit, quod elevat, quod deterret quod refutat...

(55) Cic., De Or., ibid.

(56) Cf. Plut., Apopht. Cic., 6; Cic., 26, 9; Cic. Pro Sull, 22-25; Sall., Cat., 31, 7; cf. Ps. Sall., In Cic., 1; 4 ; 7. 
Ego autem - existimes licet, quod lubet - mirifice capior facetiis, maxime nostratibus, praesertim cum eas videam primum oblitas Latio tum, cum in urbem nostram est infusa peregrinitas, nunc vero etiam braccatis et transalpinis nationibus, ut nullum veteris leporis vestigium appareat. Itaque, te cum video, omnes mihi Granios, omnes Lucilios, - vere ut dicam - Crassos quoque et Laelios videre videor (Fam., IX, 15, 2).

Mieux encore, ils ont en commun de s'attaquer à ces orateurs au latin incertain ou à l'éloquence braillarde issus souvent comme eux d'une ville d'Italie.

Q. Granius est moins connu que les deux autres (57). It était praećo, c'est-à-dire héraut de magistrat, et appartenait au cercle des amis de Crassus et de Cicéron (58). On a de bonnes raisons de penser qu'il était originaire de Pouzzoles (59). C'est lui qui, on l'a vu, l'emporte sur T. Tinca de Plaisance dans cette joute de réparties plaisantes. C'est lui aussi qui se moque d'Albius, le questeur de Mucius Scaevola (60). Dans le Pro Plancio, Cicéron en fait un exemple de franc-parler: il n'hésite pas à dire son fait à $\mathrm{P}$. Scipion Nasica et à Livius Drusus (61). Surtout, il brocarde un mauvais orateur sans aucun doute un orateur de l'eloquentia popularis - qui, à force de crier, avait perdu sa voix (62). En fin de compte, il est un des exemples les plus parfaits d'urbanitas (63).

Lucilius d'ailleurs l'apprécie tellement que: conicere in versus dictum praeconis volebam Gra$n i(64)$. Il le cite à plusieurs reprises (65) et c'est sans doute lui qui fournit à Cicéron la plupart de ses anecdotes (66). Bien qu'originaire de Suessa Aurunca (67), Lucilius aussi est aux yeux de Cicéron un modèle d'urbanitas (68). Cela ne l'empêche pas de se moquer des autres italiens qui ont la malchance de conserver leur accent natal. C'est le cas en particulier d'un Vettius au parler prénestin que l'on doit probablement identifier avec le Vettius Vettianus e Marsis que cite Cicéron (69). Il n'hésite pas enfin à

(57) Münzer, $R E$, VII, 2, nº 8, col. 1818, 1912; C. Nicolet, $O E$, II, pp. 905-906.

(58) Cf., Cic., Br., 172: familiari nostro; De Or., II, 244: familiarem vestrum; 254: tuus amicus, Crasse.

(59) Cf., Nicolet, l.c.; Wiseman, New Men, p. 234.

(60) Cic., De Or., II, 281.

(61) Pro Planc., 33.

(62) Cic., De Or., II, 282.

(63) Cf. supra.

(64) XI, 411-412 M.

(65) II, $95 \mathrm{M} ; 1181-1182 \mathrm{M}$.

(66) Cf., Br. 172; F. Marx, Lucili Carminum reliquiae, Leipzig, 1904, II, p. 44; 153 (sur Granius); N. Terzaghi, Lucilio, 1934, cf. Syme, Historia, 1964, p. 117, à propos du Gargonius de Hor. Sat., I, 2, 27; 4, 92, p. 382-383.

(67) Sur l'origine et la famille de Lucilius, cf. en dernier lieu, W. Krenkel, Lucilius Satiren, Leiden, 1970, pp. 18-29; Zur Biographie des Lucilius, ANRW, I, 2, 1972, p. 1240; J. Christes, Lucilius, ein Bericht über die Forschung, ANRW, I, 2, pp. 1185-1196 (qui donne la bibliographie antérieure et fait le point de la question); Nicolet, $O E$, II, n 204, pp. 926-929; et F. Charpin, ed. Lucilius Satires, Paris, 1978, I, pp. 8-11.

(68) Cf. Fam., IX, 15. 2; De Fin., I, 7; Cic., De Or., I, 72; II, 25.

(69) Quint., I, 5, 56 (= Lucil., 1322 M.): taceo de Tuscis et Sabinis et Praenestinis quoque (nam et eorum sermone utentem Vettium Lucilius insectatur). F. Marx, II, p. 424, identifie ce Vettius avec Vettius Philocomus, un familiaris (Suet., Gramm., 2). Il est suivi par N. Terzaghi, Lucilio, p. 398; W. Strzelecki, RE, VIIIa, ${ }^{\circ} 13$, coll. 1851-1853, 1958. C. Cichorius, Untersuchungen zu Lucilius, Berlin, 1908, p. 348-349, doute que Vettius Philocomus, sans doute un Grec, soit le Prénestin de Lucilius et renvoie à tous les autres Vettii. W. Krenkel, Zur literarischen Kritik bei Lucilius, Wiss.Z.Univ.Rostock.Ges.Sprachwiss., 7, 1957-1958, p. 280, fait le rapprochement avec Vettius Vettianus en soulignant qu'entre les Marses et les Prénestins, il n'y a pas plus de $40 \mathrm{~km}$ (cf. aussi son édition de Lucilius, II, p. 708-709). T.P. Wiseman, New Men, p. 273, pense aussi au Vettius défendu par C. Gracchus (Plut., C.Gr., 1), mais aucun indice ne vient renforcer cette hypothèse. Dans ce passage du Brutus, Cicéron est très probablement influencé par Lucilius dans sa définition de l'urbanitas: il commence par C. Titius que Lucilius citait (Macr., Sat., III, 16, 3; cf. N. Terzaghi, Lucilio, pp. 328-329) et il finit par T. Tinca et Q. Granius. Le Vettius à qui Lucilius reprochait finalement son manque d'urbanitas, a donc de bonnes chances d'être Q. Vettius Vettianus que cite Cicéron. Sur les formes dialectales de Préneste, cf., A. Ernout, Le parler de Préneste d'après les inscriptions, MSL, 13, 5, pp. 293-349. 


\section{JEAN-MICHEL DAVID}

accabler d'invectives les orateurs populaires (70).

Le cas de M. Terentius Varro est moins net, mais tout de même, on se souvient que lui aussi, natif de Rieti, n’a pas hésité, dans ses Satires Ménippées, à se moquer des rabulae, élèves de L. Plotius Gallus, le rhéteur latin (71).

Cicéron, Q. Granius, Lucilius, Varron, quatre exemples de domi nobiles venus à Rome, plus ou moins intégrés à la classe politique romaine, et qui prétendent donner à d'autres des leçons d'urbanitas. Pourquoi et comment en prennent-ils le droit?

$\mathrm{Si}$ on laisse Cicéron de côté, la première remarque que l'on peut faire est que dans les trois autres cas, les moqueries et les invectives, n'ont pas le poids de l'autorité politique. Cet humour, ces plaisanteries, ne quittent pas, le plus souvent, le niveau du discours privé, de la conversation. Les attaques de Lucilius et de Varron s'insèrent dans des satires, un genre littéraire qui reste du domaine de la critique privée des moeurs publiques (72). Le banquet y prend ainsi une place importante: c'est un banquet offert par Granius à Crassus que Lucilius veut commémorer. Au temps de Cicéron, il n’y avait plus que cette satire qui rappelât que le tribunat de Crassus avait eu lieu cette année-là, et il est très probable que les orateurs d'origine municipale y furent l'objet de multiples plaisanteries (73).

Dès lors, on comprend mieux ce que signifie l'urbanitas de Granius ou de Lucilius. C'est la courtoisie, le sens de l'humour de personnages raffinés, capables de se jouer plaisamment des ridicules d'hommes publics. Elle s'exprime au sein des cercles aristocratiques: Lucilius est un familier de Laelius et de Scipion (74); Granius est un ami de Crassus (75). Varron, lui, est lié à Pompée et à Cicéron (76). Ainsi s'opère la rencontre de la plaisanterie, du bon goût et du raffinement spécifiquement romain qui définit l'urbanitas. Elégance de banquets et de conversations privées, elle est avant tout un critère de distinction aristocratique.

Dans un commentaire à la lettre de Cicéron à Paetus que l'on a citée tout à l'heure, J.H. D'Arms

(70) VI, 261-262: haec, inquam, rudet ex rostris atque heiulitabit, concursans, veluti Ancarius, clareque quiritans, cf. F. Marx, ed. Lucilius, II, p. 99; C. Cichorius, Untersuchungen, pp. 281-286, qui identifie ce personnage avec C. Memmius, le tribun de 111; N. Terzaghi, Lucilio, p. 346; Krenkel,-ed. Lucilius, I, pp. 200-201.

(71) Sat. Men., p. 186, 6 R = Papiapapae XI; 157 R; cf. MEFRA, 91, 1979, p. 157; L. Deschamps, L'attitude de Varron face à la rhétorique dans les satires Ménippées, Scritti in onore di B. Riposati, Milan, 1979, I, pp. 157-166. Sur Varron, cf. en dernier lieu, A. Garzetti, Varrone nel suo tempo, Atti Congr. intern. Studi Varroniani, Rieti, 1974, Rieti, 1976, I, pp. 91-110.

(72) U. Knoche, Die römische Satire, Göttingen, 1957, p. 21; W. Krenkel, Römische Satire und römische Gesellschaft, Wiss. Z. Univ. Rostock. Ges. Sprachwiss., 15, 1966, pp. 471-477; E.G. Schmidt, Diatribe und Satire, ibid., pp. 507-515; E.S. Ramage, D.L. Sigsbee, S.C. Fredericks, Roman Satirists and their Satire, the fine art of criticism in ancient Rome, Park Ridge, 1974, pp. 31-32.

(73) Cic., Br., 160; cf. N. Terzaghi, Lucilio, pp. 7; 324-336; J. Martin, Symposion, Die Geschichte einer literarischen Form, Paderborn, 1931, pp. 214-216. F. Marx, ed. Lucilius, I, p. XLIX; 39 (suivi par W. Krenkel, ed. Lucilius, pp. 332-333; F. Charpin, ed. Lucilius, Paris, 1979, II, pp. 105-106, mais avec une erreur p. 263) identifie les quelques fragments du livre XX avec la description de ce repas. Il pense, en effet, très justement que c'est à Licinius Crassus qu'il faut attribuer les mots de rage à l'égard de la lex Calpurnia puisqu'en 106, l'année suivante, il soutenait la lex Servilia Caepionis en s'opposant aux accusateurs assoiffés de sang (MÉFRA, 91, 1979, pp. 142-143; 165; 167-168). Il est très tentant d'imaginer qu'au cours de ce banquet ait été mise en scène une satire des accusateurs et orateurs populaires d'origine municipale qui aurait fourni à Cicéron les principaux matériaux du Brutus et du De Oratore. Les vers 575-576 contiennent une allusion - bien peu claire, il est vrai- à un Marse magicien et c'est peut-être là que se seraient trouvés les vers 1174-1176 qui sont la citation de C. Titius (cf. Marx ad loc. et supra n. 69). On comprendrait ainsi la spontanéité avec laquelle Cicéron cite dans le Brutus, 160, le rôle de Crassus dans la lex Servilia Caepionis, tout de suite après l'évocation de ce banquet.

(74) Cf. en dernier lieu, B. Zucchelli, L'indipendenza di Lucilio, Florence, 1977.

(75) Cf. supra pag. 319 et n. 57.

(76) A. Garzetti, o.c., cf. aussi, bien que très générales, les remarques d'H. Bardon, La notion d'intellectuel à Rome, Stud.Class., 13, 1971, pp. 96-101. 


\section{LES ORATEURS DES MUNICIPES A ROME}

remarquait naguère que tous ces personnages que Cicéron présentait comme des modèles d'urbanitas, étaient liés à la Campanie (77). Il imaginait ainsi que cette lettre était une allusion à la tradition régionale de l'humour campanien. En fait si la Campanie est un haut lieu de l'urbanitas, c'est parce qu'elle est un des sites préférés de villégiature des sénateurs romains. Comme autrefois la Normandie et Paris, la baie de Naples et Rome sont les deux centres privilégiés de l'élégance et du raffinement. Des hommes comme Q. Granius et Lucilius, originaires de la région, peuvent s'insérer dans le réseau clientélaire de cette aristocratie et être admis à ses mondanités. Certes, on ne mettra pas les deux hommes sur le même plan. Lucilius est un chevalier romain et d'un rang social finalement très proche de celui de ses amis. Mais le mécanisme est le même. Les cercles aristocratiques reçoivent en leur sein des personnages issus de l'élite locale de Pouzzoles ou de Suessa Aurunca qui doivent pour être admis perdre tout accent, tout soupçon de rusticité. Nul doute que ces domi nobiles n'effacent tout stigmate, toute trace de non-romanité (78). En reconnaissant symboliquement la supériorité des normes culturelles dont ils ne sont pas les détenteurs légitimes, ils se placent ainsi dans une position subordonnée et révérencieuse (79). La récompense est le droit de se mêler à cette sociabilité toute privée de la conversation savante et raffinée. Le banquet, avec son jeu de réparties et de plaisanteries élégantes en est un des temps forts. C'est là en effet que la fausse égalité de rapports amicaux autorise d'autant plus les critiques et le franc-parler qu'ils sont socialement et politiquement inoffensifs.

Cette procédure de déculturation et d'acculturátion est aussi un mode d'intégration et de contrôle, car le prix à payer de ce droit à la plaisanterie privée est une exclusion de fait de la parole publique.

Bien qu'un peu particulier, le cas de Q. Granius est parfaitement net. Le rôle social d'un praeco est en effet d'être le porte-parole du magistrat qui l'emploie. Muet politique, ses discours sont condamnés à l'inefficacité et à l'irresponsabilité. Il ne faut pas s'étonner qu'en échange de son exclusion de la sphère publique, il ait pu —sorte de fou du roi-s'autoriser une liberté de parole qu'on lui contestait d'autant moins qu'elle avait peu d'importance. Comme la plupart de ses collègues, il revalorisait par la boutade ou le bon mot, une voix mutilée par la perte de sa valeur publique (80).

De la même façon, les conversations et plaisanteries qui s'échangent dans les cercles aristocratiques restent sans effet politique. Cette sociabilité-là inverse souvent les valeurs de la sociabilité publique. Le banquet surtout est un moment de forte transgression des normes civiques (81). Il oppose au modèle public de frugalité et de tempérance qui caractérise la gravitas aristocratique, la consommation raffinée et ludique de vins et de mets travestis. En défi aux règles du mariage, il autorise les jeux érotiques de l'homosexualité. Les règles qu'il édicte pour son organisation propre, parodient les lois de la cité. Rien de tout ceci n'est innocent. Ces violations rituelles du code

(77) Two passages from Cicero's Correspondance, AJPh, 88, 1967, pp. 200-202; et, d'une façon générale, Romans on the bay of Naples, Cambridge Mass., 1970.

(78) E.S. Ramage, $A J P h, 84,1963$, p. 398 ; Urbanitas, p. 57; remarque que la notion d'urbanitas passe pour Cicéron par la vie à Rome et l'association avec des romains raffinés.

(79) Dans un article éclairant, A. Pennacini, Docti e crassi nella poetica de Lucilio, A.Acc.S.Torino, 1965-1966, pp. 293-360, a montré que Lucilius prétendait viser un public intermédiaire entre les doctissimi aristocrates et les indoctissimi populaires et ouvrir la communication à une aire sociale et ethnique qui aurait compris Romains et Italiens. Lucilius se plaçait donc dans une position où il échangeait la reconnaissance de la supériorité aristocratique contre le droit d'être distingué des classes populaires. Il espérait y intégrer avec lui ses compagnons de classe sociale, domi nobiles et classes intermédiaires de Rome (cf. ceux qu'il cite comme son public: les Tarentins, les Consentins, les Siciliens, M. Iunius Congus et D. Laelius qui ne sont sénateurs ni l'un ni l'autre, peut-être chevaliers, mais rien ne le prouve).

(80) Cf. Mommsen, Dr. Pub., I, pp. 416-420 et surtout F. Hinard, Remarques sur les praecones et le praeconium dans la Rome de la fin de la République, Latomus, 35, 1976, pp. 730-746, en part. p. 733 et pp. 742-746: on doit ajouter que l'infamie qui les frappe tient certainement à cette mutilation symbolique (cf. l'image de la prostitution de la voix, Cic., Pro Quinct., $13 ; 95)$.

(81) Cf. J. Martin, Symposion, et surtout, F. Dupont, Le plaisir et la loi, Paris, 1977. 
officiel sont la condition même de la vie pólitique. Fête ou carnaval permanent d'une aristocratie soumise aux fortes tensions d'une compétition sans pitié, il libère des frustrations qu'engendre l'obligation d'un comportement conforme à la tradition familiale et civique.

On voit bien ainsi comment un nouveau venu admis à ces jeux, peut s'y enfermer et perdre tout espoir de carrière autonome. Les qualités d'élégance mondaine dont il est obligé de faire preuve ne lui sont d'aucun secours dans les conflits politiques. Au contraire même, les transgressions auxquelles il est parfois contraint dans une surenchère coûteuse, le desservent. Les efforts qu'il.fait pour être admis à la compagnie des grands, l'enfoncent de plus en plus dans la dépendance clientélaire.

Il y a donc, pour un domi nobilis, une sorte de contradiction entre l'intégration aux cercles aristocratiques et la reconnaissance publique que le cas de Valerius Valentinus illustre assez bien. Ce personnage est originaire de Vibo Valentia, une ville de droit latin, en Italie du Sud. Il se mêle à l'entourage de L. Opimius, le consul de 121, l'ennemi des Gracques. Là, en parfaite conformité avec les règles de la sociabilité aristocratique, il compose des vers et même une loi de banquet: la lex Tappula, une parodie des lois populares, dont on a la chance d'avoir conservé une copie épigraphique. Plus tard, son patron est exilé et il tente sa chance au jeu de la promotion civique. Vers $105-95$, il accuse de repetundis, un certain C. Cosconius. Sans doute espérait-il obtenir la citoyenneté romaine. C'est un échec car son adversaire cite de lui des vers érotiques qu'il avait composés naguère. La place qu'il avait tenue dans le cercle d'Opimius, ses transgressions des normes sociales, lui interdisent l'accès au statut civique qu'il prétendait obtenir (82).

Deux voies s'ouvrent donc aux domi nobiles italiens soucieux de s'intégrer à la classe politique romaine. La première est celle de l'admission au sein des clientèles aristocratiques et de la progression dans l'amitié d'un grand. Elle exige des qualités d'urbanitas, d'élégance et de raffinement. Certes, elle n'interdit pas que l'on mène une carrière politique, mais elle la subordonne étroitement à l'appui et à la reconnaissance active de l'aristocrate auquel on s'est lié. A partir de la fin du IIème siècle, de nouvelles filières apparaissent qui permettent de gagner directement la confiance populaire; l'accusation devant les iudicia publica, l'exercice du tribunat de la plèbe sont de celles-là. Elles passent par l'emploi d'une éloquence violente faite d'appels à l'émotion et au pathétique. On comprend que, surtout dans les années qui précèdent la guerre sociale, certains de ceux qui ont payé l'intégration aux cercles aristocratiques d'un renoncement à la politique, perçoivent comme une usurpation la promotion rapide d'orateurs d'origine municipale à peine formés à une rhétorique de l'efficacité immédiate. Des hommes comme Granius et Lucilius sont ainsi parmi les premiers à railler et à invectiver ces nouveaux venus, à leur refuser le bénéfice d'une reconnaissance sociale trop facilement gagnée. Après la guerre sociale, le mouvement d'unification juridique, politique et culturelle de l'Italie enlève sans doute de son acuité au problème. Il persiste néanmoins. Cicéron d'Arpinum n'hésite pas à reprocher leur physique ou leur naissance à certains de ses adversaires, comme lui, d'origine municipale. Lui qui s'est formé au contact de L. Licinius Crassus et de M. Antonius, et a hérité de leur dégoût pour une formation hâtive, n'accepte pas que l'on puisse ne pas respecter le modèle de comportement aristocratique. Bien sûr, lui aussi accuse et cherche à se rendre populaire. Mais il ne le fait que tardivement, après une longue période de formation et de plaidoieries modestes. Il respecte les étapes d'une intégration progressive et méritante dans la classe politique (83). Il ne supporte pas que d'autres brûlent les étapes qu'il a dû respecter.

En somme, l'intégration des notables locaux dans la classe politique romaine n'est pas une chose facile. Celui qui emprunte la voie disons "marienne" des honneurs que l'on obtient directement du

(82) Cf. MÉFRA, 91, 1979, pp. 151-152; 180; et aussi, B. Zucchelli, L'indipendenza di Lucilio, p. 115, n. 112.

(83) Cf. E. Rawson, Homo novus Arpinas ex M. Crassi familia, [Sallust], Cic., 3, LCM, 1, 1976, pp. 93-95, qui modifie M. en L. L'auteur de l'invective reproche donc à Cicéron à la fois son origine et son intégration clientélaire. 


\section{LES ORATEURS DES MUNICIPES A ROME}

peuple en s'appuyant sur ses aspirations et sa sensibilité, rencontre l'hostilité de la noblesse. Celui qui au contraire, emprunte la voie "lucilienne" de l'admission aux cercles aristocratiques, s'enferme dans des relations clientélaires qui le subordonnent aux grands. Il ne peut accéder au pouvoir qu'autant qu'ils le permettent. Certes, il n'est pas exclu que l'on puisse, comme Cicéron, emprunter l'une et l'autre en essayant de conserver une relative indépendance: c'est même sans doute là, la pratique la plus générale, surtout quand, après la guerre sociale, l'Italie s'unifie. Il n'en reste pas moins que ces deux pôles de l'exclusion ou de l'intégration, comme on voudra, demeurent. Ils sont l'image de l'illégitimité de la promotion sociale. Valerius de Sora ou Gargonius de Bologne pourront être des modèles de gravitas ou de frugalité, ils resteront des paysans. Valerius Valentinus est un poète: ses vers lui valent de ne pas être jugé digne de défendre la cité. Un noble n'a pas à craindre de telles mésaventures. Sa légitimité le met au-dessus de la censure: pour lui, transgresser une norme, c'est en fonder une nouvelle. 